\title{
Warfarin plus Aspirin or Aspirin Alone for Patients with Giant Coronary Artery Aneurysms Secondary to Kawasaki Disease?
}

\author{
Michael Levin ${ }^{a}$ Jane C. Burns ${ }^{b, d}$ John B. Gordon ${ }^{c, e}$ \\ a Section for Paediatrics, Division of Medicine, Imperial College London, London, UK; Departments of bPediatrics and \\ 'Medicine, University of California San Diego School of Medicine, dRady Children's Hospital, and 'San Diego Cardiac \\ Center, San Diego, Calif., USA
}

Kawasaki disease is an acute inflammatory disorder predominantly affecting young children. Since its initial description by Dr. Kawasaki in children in Japan, the disease has emerged as a relatively common childhood condition with an incidence varying from 240/100,000 children aged under 5 years in Japan to 8-19/100,000 in the USA and Europe [1,2]. Although epidemiological features strongly suggest an infectious aetiology, the cause of Kawasaki disease remains unknown, and current thinking suggests that the disease is triggered by one or more as yet unidentified pathogens or their toxins, which initiate an inflammatory process in genetically predisposed individuals [3].

Prior to the introduction of intravenous immunoglobulin, which is now the standard recommended treatment, $20-30 \%$ of children with Kawasaki disease developed coronary artery aneurysms (CAAs) [4]. Although intravenous immunoglobulin reduces the risk of CAAs to approximately $5-10 \%$, a significant proportion of affected children fail to respond to immunoglobulin or are treated too late to prevent coronary artery damage. For those patients who fail to respond to intravenous immunoglobulin there is growing evidence that steroids or other antiinflammatory agents, such as infliximab, anakinra or cyclosporine, may be effective in controlling the in-

\section{KARGER}

E-Mail karger@karger.com

www.karger.com/crd flammatory process and reducing coronary artery damage [5-7]. However, despite the growing armamentarium of anti-inflammatory treatments, $5-10 \%$ of patients with Kawasaki disease are left with coronary artery injury, ranging from mild dilatation to severe and persistent aneurysms [8].

Mid-term follow-up into the second and third decade of life of children who have had normal echocardiograms provides reassurance that these young adults have no observable cardiovascular sequelae of their Kawasaki disease [9]. Follow-up of patients who suffered coronary artery injury as manifested by CAAs measuring less than 10 standard deviations from the mean normalized for body surface area ( $\mathrm{Z}$ score) is in progress and the outcomes are still being defined [10]. However, for those patients who have developed giant CAAs (with diameters in excess of $8 \mathrm{~mm}$ ) the long-term outcome is extremely worrying [1113]. In a review of 245 patients with giant aneurysms, Tsuda et al. [11] reported that $60 \%$ of children followed for a median of 20 years after the onset of Kawasaki disease required coronary artery interventions, such as angioplasty, coronary artery bypass grafting or cardiac transplantation; $10 \%$ died and only a third remained well without either suffering a myocardial infarct or requiring cardiac intervention. Considering that most children
(C) 2014 S. Karger AG Basel

$0008-6312 / 14 / 1293-0174 \$ 39.50 / 0$
Michael Levin, FRCPCH, FMedSci

Section for Paediatrics, Division of Medicine

Imperial College London, Norfolk Place

London W2 1PG (UK)

E-Mail m.levin@imperial.ac.uk 
with Kawasaki disease are less than 5 years old at the time of the illness, even 20 years of follow-up only provides information into early adult life, and there is considerable concern that longer follow-up will show continued attrition, with early death or myocardial infarction of an even greater proportion of those with giant aneurysms [14]. As the numbers of children who have suffered severe coronary artery injury as a result of Kawasaki disease continues to increase, and children with persistent CAAs progress to adulthood, adult cardiologists and physicians, as well as paediatricians, are faced with the dilemma of how best to manage patients with giant CAAs who remain at high risk of acute coronary artery thrombosis and myocardial ischaemia throughout life.

Two major processes appear to be responsible for the poor cardiac outcome in children with giant CAAs. Firstly, the perturbed haemodynamics with reduced wall sheer stress and increased particle residence time lead to a significant risk of thrombotic occlusion of the aneurysm lumen and of fragments of thrombi embolising distally to occlude smaller vessels downstream of the aneurysm [15]. Secondly, intimal thickening due to luminal myofibroblastic proliferation and calcification of layered thrombus in regions of turbulent flow at the junctions between the aneurysm and the more normal calibre artery may result in stenosis and consequently myocardial ischaemia occurring many years after the acute illness [16].

Management of children with giant CAAs has focused on how best to prevent both thrombosis within the aneurysm and the process of vascular remodelling that leads to stenotic lesions. It should be made clear that vasculopathy and atherosclerotic changes are two distinct pathophysiologies [16]. There is currently no evidence to suggest that children who suffer giant aneurysms are at increased risk of atherosclerotic changes in the regions of the aneurysms. In fact, there is anecdotal evidence to the contrary. However, until more data are available, it seems prudent to counsel patients about general atherosclerosis risk reduction. Hyperlipidaemia, hypertension and obesity should be aggressively managed. Tobacco use should be discouraged. The potential benefit of statins in this population should be considered, not so much for the lipid-lowering effects, but more for the anti-inflammatory and anti-remodeling benefits $[17,18]$. However, in addition to these generic interventions, there has been considerable debate as to whether patients with giant CAAs should be treated with anti-platelet agents alone or with a combination of anti-platelet agents and anti-coagulation with warfarin [4].

Warfarin plus Aspirin or Aspirin Alone?
The decision to commence a young child on anti-coagulation is a difficult one that requires balancing the potential benefit of reducing the risk of thrombosis within the aneurysm against the effect on the lifestyle of the child and their family imposed by treatment with a drug requiring frequent laboratory monitoring, and which carries a risk of bleeding. The risks of bleeding following trauma, while on anti-coagulant treatment, may be greater for children than for adults. In the toddler years, children fall and have frequent head bangs as an inevitable part of development. In the younger school years, falls and collisions occur frequently during play, and in the teenage years, risky adolescent behaviour and activities, such as cycling, rollerblading, skiing or martial arts, all carry a considerable risk of internal bleeding for patients on anticoagulation. Although parents and health professionals may advise children with giant CAA who are on warfarin to avoid contact sports and other risky activities, such advice is frequently ignored by teenagers and young adults and is impossible to enforce. Clinicians caring for children with giant CAA are thus in need of clear evidence to support the use of anti-coagulants, particularly in view of the considerable risks of bleeding and the disruption of life imposed by anti-coagulation.

Ideally a decision to commence a child with giant CAA on warfarin should be based on evidence from randomised, placebo-controlled trials. However, due to the relative rarity of giant CAA, and the life-threatening consequences of thrombosis within the aneurysm, it is not surprising that randomised trials comparing anti-coagulation with warfarin with the use of anti-platelet agents alone have not been undertaken. In the absence of randomised controlled trials, clinical practice has been guided by the results of non-randomised long-term follow-up studies, several of which have suggested that anti-coagulation with warfarin carries a lower risk of fatal outcome, aneurysm thrombosis or the need for coronary artery interventions compared to treatment with aspirin alone $[11,19]$. It is fair to say that there is no longer clinical equipoise on this matter and current national guidelines from Japan and the USA advocate this treatment $[4,20]$.

In a recent issue of Cardiology, Su et al. [21] report a meta-analysis of the published long-term follow-up studies of children treated with anti-coagulation therapy and aspirin, or aspirin alone. They conducted a systematic search of PubMed, the Cochrane library, EMBASE, Science Citation, Web of Science and Chinese Biomedicine literature databases to identify studies reporting longterm follow-up of children with CAAs. They identified 324 articles relating to aspirin or warfarin treatment. Af- 
ter excluding case reports, abstracts and studies not meeting their criteria for review, only 6 were found eligible for inclusion, all of which were retrospective, non-randomised, case-controlled trials published between 2005 and 2013. The trials provided data on 159 children with giant CAAs secondary to Kawasaki disease who were treated with warfarin plus aspirin, and 158 children who were treated with aspirin alone. There was considerable heterogeneity between these studies in the timing of introduction of warfarin, and in the total duration of follow-up.

The meta-analysis demonstrated a significantly lower incidence of death in children with giant CAA treated with warfarin plus aspirin as compared to those treated with aspirin alone (odds ratio $0.18,95 \%$ confidence interval $0.04-0.88, p=0.03)$. Furthermore, there was a significantly lower incidence of coronary artery occlusion in patients treated with warfarin plus aspirin as compared to those treated with aspirin alone (odds ratio 0.08, 95\% confidence interval $0.02-0.29, \mathrm{p}=0.001$ ), and a significantly lower incidence of myocardial infarction (odds ratio $0.27,95 \%$ confidence interval $0.11-0.63, p=003$ ). No difference was observed between those patients treated with warfarin and aspirin and those treated with aspirin alone in the rate of regression of CAAs, in the incidence of persistent CAAs, or in the incidence of coronary artery stenosis. Surprisingly, the meta-analysis did not reveal any significant difference in the incidence of thrombus formation within the giant CAA. This raises the question of whether warfarin is an imperfect treatment for this indication given that the target international normalised ratio is achieved only two thirds of the time in the best of circumstances [22]. The papers included in the metaanalysis contained only sparse data on haemorrhagic complications. In one of the trials, 8 cases of nasal bleeding and 1 case of subarachnoid haemorrhage occurred in children treated with warfarin plus aspirin, while only 3 cases of nasal bleeding and no severe haemorrhagic complications occurred in those on aspirin. In a second study, $80 \%$ of children with giant CAA treated with warfarin plus aspirin experienced a significant haemorrhage at least once during treatment. In a third study, 6 of 71 children treated with warfarin plus aspirin experienced haemorrhage, and in one study there were no cases of severe haemorrhage in either group.

This meta-analysis of published long-term follow-up studies of children with giant CAA treated with warfarin and aspirin or aspirin alone provides the best data currently available to support the long-term use of anti-coagulation in children with giant CAAs. A surprising find- ing was that no differences were observed between warfarin plus aspirin and aspirin alonein thrombus formation, whereas there were significant differences in the rates of myocardial infarction and death, which were both reduced in the warfarin-treated group. It is unlikely that warfarin would have achieved the beneficial effect on coronary artery occlusion, myocardial infarction and death through any other effect other than its action as an anticoagulant. The finding of no effect on the rate of thrombus formation is most likely due to clinical difficulty in distinguishing myocardial ischaemia and infarction as a result of thrombosis and embolisation from that caused by stenosis of the artery.

As all the studies included in the meta-analysis were non-randomised case-control follow-up studies, there is likely to be some bias in selection of patients for treatment with warfarin plus aspirin or aspirin alone. However, given the significant benefit in terms of death and myocardial ischaemic events in the warfarin-treated patients, it would be hard to now justify a randomised or placebocontrolled trial. The main conclusion of this meta-analysis is that patients with giant CAA are likely to benefit from anti-coagulation as well as anti-platelet therapy despite the risks and difficulties in maintaining long-term anti-coagulation throughout childhood.

While this meta-analysis provides strong support for anti-coagulation as well as aspirin for children with giant CAA, it should also stimulate further trials comparing new oral anti-coagulant agents against warfarin. There is growing evidence in adult practice that the new anti-coagulant drugs, such as dabigatran, apixaban and rivaroxaban, are effective in reducing the risk of stroke in patients with atrial fibrillation, and in the treatment of deep vein thrombosis and pulmonary embolization [23]. These agents have several advantages over warfarin, particularly in their ease of administration and lack of need for frequent blood monitoring. However, there is concern that they are less easy to reverse in the event of bleeding and their safety during long-term administration, particularly in children, has yet to be established. Given that a child commenced on anti-coagulation in the early years of childhood may require anti-coagulation for many decades, long-term safety information and experience in the use of these agents in children may be required before they can be considered as alternatives to warfarin.

Based on studies reviewed in this meta-analysis, and the significant benefit in terms of reduced myocardial infarction and death, long-term oral anti-coagulation with warfarin together with aspirin appears to be the current best management of children with giant CAAs. 


\section{References}

-1 Nakamura Y, Yashiro M, Uehara R, Sadakane A, Tsuboi S, Aoyama Y, Kotani K, Tsogzolbaatar EO, Yanagawa H: Epidemiologic features of Kawasaki disease in Japan: results of the 2009-2010 nationwide survey. J Epidemiol 2012;22:216-221.

-2 Uehara R, Belay ED: Epidemiology of Kawasaki disease in Asia, Europe, and the United States. J Epidemiol 2012;22:79-85.

$\checkmark 3$ Rowley AH: Kawasaki disease: novel insights into etiology and genetic susceptibility. Ann Rev Med 2011;62:69-77.

-4 Newburger JW, Takahashi M, Gerber MA, Gewitz MH, Tani LY, Burns JC, Shulman ST, Bolger AF, Ferrieri P, Baltimore RS, Wilson WR, Baddour LM, Levison ME, Pallasch TJ, Falace DA, Taubert KA: Diagnosis, treatment, and long-term management of Kawasaki disease: a statement for health professionals from the Committee on Rheumatic Fever, Endocarditis and Kawasaki Disease, Council on Cardiovascular Disease in the Young, American Heart Association. Circulation 2004;110:2747-2771.

5 Kobayashi T, Morikawa A, Ikeda K, Seki M, Shimoyama S, Ishii Y, Suzuki T, Nakajima K, Sakamoto N, Arakawa H: Efficacy of intravenous immunoglobulin combined with prednisolone following resistance to initial intravenous immunoglobulin treatment of acute Kawasaki disease. J Pediatr 2013;163:521526.

6 Son MB, Gauvreau K, Burns JC, Corinaldesi E, Tremoulet AH, Watson VE, Baker A, Fulton DR, Sundel RP, Newburger JW: Infliximab for intravenous immunoglobulin resistance in Kawasaki disease: a retrospective study. J Pediatr 2011;158:644-649.e1.

$\checkmark 7$ Tremoulet AH, Pancoast P, Franco A, Bujold M, Shimizu C, Onouchi Y, Tamamoto A, Erdem G, Dodd D, Burns JC: Calcineurin inhibitor treatment of intravenous immunoglobulin-resistant Kawasaki disease. J Pediatr 2012;161:506-512.e1.
$>8$ Ogata S, Tremoulet AH, Sato Y, Ueda K, Shimizu C, Sun X, Jain S, Silverstein L, Baker AL, Tanaka N, Ogihara Y, Ikehara S, Takatsuki S, Sakamoto N, Kobayashi T, Fuse S, Matsubara T, Ishii M, Saji T, Newburger JW, Burns JC: Coronary artery outcomes among children with Kawasaki disease in the United States and Japan. Int J Cardiol 2013;168:3825-3828.

$\checkmark 9$ Holve TJ, Patel A, Chau Q, Marks AR, Meadows A, Zaroff JG: Long-term cardiovascular outcomes in survivors of Kawasaki disease. Pediatrics 2014;133:e305-e311.

10 Manlhiot C, Millar K, Golding F, McCrindle BW: Improved classification of coronary artery abnormalities based only on coronary artery z-scores after Kawasaki disease. Pediatr Cardiol 2010;31:242-249.

-11 Tsuda E, Hamaoka K, Suzuki H, Sakazaki H, Murakami Y, Nakagawa M, Takasugi H, Yoshibayashi M: A survey of the 3-decade outcome for patients with giant aneurysms caused by Kawasaki disease. Am Heart J 2014; 167:249-258.

-12 Sugahara Y, Ishii M, Muta H, Iemura M, Matsuishi T, Kato H: Warfarin therapy for giant aneurysm prevents myocardial infarction in Kawasaki disease. Pediatr Cardiol 2008;29: 398-401.

13 Suda K, Iemura M, Nishiono H, Teramachi Y, Koteda Y, Kishimoto S, Kudo Y, Itoh S, Ishii H, Ueno T, Tashiro T, Nobuyoshi M, Kato H, Matsuishi T: Long-term prognosis of patients with Kawasaki disease complicated by giant coronary aneurysms: a single-institution experience. Circulation 2011;123:1836-1842.

14 Nakamura Y, Aso E, Yashiro M, Tsuboi S, Kojo T, Aoyama Y, Kotani K, Uehara R, Yanagawa H: Mortality among Japanese with a history of Kawasaki disease: results at the end of 2009. J Epidemiol 2013;23:429-434.

- 15 Sengupta D, Kahn AM, Kung E, Esmaily Moghadam M, Shirinsky O, Lyskina GA, Burns JC, Marsden AL: Thrombotic risk stratification using computational modeling in patients with coronary artery aneurysms following Kawasaki disease. Biomech Model Mechanobiol 2014, Epub ahead of print.
16 Orenstein JM, Shulman ST, Fox LM, Baker SC, Takahashi M, Bhatti TR, Russo PA, Mierau GW, de Chadarevian JP, Perlman EJ, Trevenen C, Rotta AT, Kalelkar MB, Rowley $\mathrm{AH}$ : Three linked vasculopathic processes characterize Kawasaki disease: a light and transmission electron microscopic study. PLoS One 2012; 7:e38998.

17 Blankier S, McCrindle BW, Ito S, Yeung RS: The role of atorvastatin in regulating the immune response leading to vascular damage in a model of Kawasaki disease. Clin Exp Immunol 2011;164:193-201.

18 Niedra E, Chahal N, Manlhiot C, Yeung RS, McCrindle BW: Atorvastatin safety in Kawasaki disease patients with coronary artery aneurysms. Pediatr Cardiol 2014;35:89-92.

19 Suda K, Kudo Y, Higaki T, Nomura Y, Miura M, Matsumura M, Ayusawa M, Ogawa S, Matsuishi T: Multicenter and retrospective case study of warfarin and aspirin combination therapy in patients with giant coronary aneurysms caused by Kawasaki disease. Circ J 2009;73:1319-1323.

20 Research Committee of the Japanese Society of Pediatric Cardiology, Cardiac Surgery Committee for Development of Guidelines for Medical Treatment of Acute Kawasaki Disease: Guidelines for medical treatment of acute Kawasaki disease: report of the Research Committee of the Japanese Society of Pediatric Cardiology and Cardiac Surgery (2012 revised version). Pediatr Int 2014;56: 135-158.

21 Su D, Wang K, Qin S, Pang Y: Safety and efficacy of warfarin plus aspirin combination therapy for giant coronary artery aneurysm secondary to Kawasaki disease: a meta-analysis. Cardiology 2014;129:55-64.

22 van Walraven C, Jennings A, Oake N, Fergusson D, Forster AJ: Effect of study setting on anticoagulation control: a systematic review and metaregression. Chest 2006;129:11551166.

23 Schulman S: New oral anticoagulant agents general features and outcomes in subsets of patients. Thromb Haemost 2014;111:575-582. 